Research Paper

\title{
Global Analysis of miRNA Signature Differentially Expressed in Insulin-resistant Human Hepatocellular Carcinoma Cell Line
}

\author{
Linjing $\mathrm{Li}^{1 * \bowtie}$, Yan Cheng ${ }^{2 *}$, Li Lin ${ }^{3^{*}}$, Zhuan Liu ${ }^{1}$, Shengfang Du ${ }^{4}$, Li Ma ${ }^{1}$, Jing Li ${ }^{1}$, Zhiheng Peng ${ }^{1}$, Jing Yan ${ }^{1}$ \\ 1. Department of Clinical Laboratory Center, The Second Hospital of Lanzhou University, Lanzhou 730000, P.R. China. \\ 2. Northwest University for Nationalities, Lanzhou 730000, P.R. China. \\ 3. Hematology Department, Gansu Provincial Cancer Hospital, Lanzhou, Gansu 730000, China. \\ 4. Department of Anesthesiology, the Second Hospital of Lanzhou University, Lanzhou 730000, P.R. China. \\ *These authors contributed equally to this article. \\ $\triangle$ Corresponding author: Linjing Li, Department of Clinical Laboratory Center, The Second Hospital of Lanzhou University, 82 Cuiying Gate Road, Lanzhou, \\ Gansu, 730000, P.R. China, E-mail: lilinj@lzu.edu.cn.
}

(c) The author(s). This is an open access article distributed under the terms of the Creative Commons Attribution License (https://creativecommons.org/licenses/by/4.0/). See http:/ /ivyspring.com/terms for full terms and conditions.

Received: 2019.11.11; Accepted: 2020.02.21; Published: 2020.02.24

\begin{abstract}
Chemoresistance mediated by insulin resistance (IR) in HCC has already been validated. However, the underlying mechanism, especially the involvement of microRNAs (miRNAs) was unelucidated. In this study, miRNA microarrays and bioinformatics methods were employed to determine the dysregulation of miRNA by IR in HCC cells, and quantitative RT-PCR (qRT-PCR) was applied to valid the miRNA array data. Of all the 2006 miRNAs screened, 32 miRNAs were found up or down regulated between the HepG2/IR cells and its parental cells. Further literature mining revealed that some of these miRNAs may function as oncogenes or tumor suppressors that contribute to tumor progression, recurrence, and metastasis which eventually lead to chemotherapeutic resistance. Interestingly, bioinformatics analysis by Gene Ontology (GO) enrichment pathway indicating that function of the predicted target genes of these dysregulated miRNAs were significantly enriched in the processes related with biosynthesis, catabolism, modification etc., and Kyoto Encyclopedia of Genes and Genomes (KEGG) mapping showed that the biological regulatory mechanisms were integrated in cancer-related pathways. Moreover, we also constructed a network which connected the differentially expressed miRNAs to target genes, GO enrichments and KEGG pathways to reveal the hub miRNAs, genes and pathways. Collectively, our present study demonstrated the possible miRNAs and predicted target genes involving in the pathophysiology of insulin resistant HCC, providing novel insights into the molecular mechanisms of multidrug resistance in the insulin resistant HepG2 cells.
\end{abstract}

Key words: insulin resistance, HCC, miRNA Signature

\section{Introduction}

Hepatocellular carcinoma (HCC) is one of the most common cancers with high mortality rates worldwide [1]. Unfortunately, some HCC patients are not eligible for radiofrequency ablation or surgical resections, thus must only rely on chemotherapy. However, the inherent multidrug resistance (MDR) towards chemotherapeutics often impairs the effect of chemotherapy for HCC therefore cannot produce satisfactory clinical outcomes [2]. Drug resistance is a multifactorial phenomenon involving many mechanisms, such as T2DM and IR caused by tumorigenesis and inflammation in the liver [3-5]. Our previous studies also validated that IR enhances the tolerance of HCC cells to chemotherapeutic [6-9], indicating that IR contributes to the inherent MDR of HCC. Nevertheless, the key underlying mechanism of the 
acquisition of resistance to chemotherapeutic drugs still remain largely unexplored.

miRNAs are endogenously expressed small noncoding RNAs with 18-25 nucleotides which play a vital role in the regulation of gene expression at posttranscriptional level [10]. Emerging evidences have revealed that miRNAs are important modulators in many signaling pathways involved in tumorigenesis [11]. Furthermore, significant variation with miRNA expression were observed in drug tolerant cancer cells in comparison with their parental drug sensitive cancer cells which displays as higher expression of cancer-promoting miRNAs (oncomiRNAs) and lower expression of cancer-inhibiting miRNAs (tumor suppressor miRNAs) [12].They might serve as tumor suppressors or oncogenes and constitute ideal targets in exploring anticancer therapeutics [13]. miRNAs have been showed as regulators that can promote or impair drug resistance in several cancers including HCC. Kabir TD et al reported that miR-7 could overcome sorafenib resistance by suppressing its target TYRO3 via PI3-Kinase/AKT pathway [14]. Xia H etc. declared that miR-216a/217 could activate TGF- $\beta$ pathway to induce sorafenib resistance [15]. Meng $W$ etc. believed that inhibition of the expression of miR-33a-5p could reduce cisplatin sensitivity and increased its drug resistance in HCC [16]. Another group identified the role of miR-195 in developing drug resistance in HCC cell line [17]. They found that it might improve 5-FU sensitivity by targeting Bcl-w protein to increase cell apoptosis. It was also reported that miR-3129-5p and its target gene Zeb1 endow HCC cells a tolerance to doxorubicin [18]. Due to the important role of miRNAs in the development of drug resistance of HCC, it is useful to employed global and systematic analytic techniques to assess the miRNA expression profiles in the drug resistant HCC cells.

Chemoresistance mediated by insulin resistance in HCC have already been validated by our previous studies [6-9], while little was known concerning the role of miRNAs in IR-mediated chemotherapy resistance. Thus, in the current study, comprehensive expression profiling of miRNAs by microarray was performed in insulin-resistant and parental HCC cell lines. Differentially expressed miRNA were identified between these two cell lines with bioinformatics analyses, which may contribute to a better understanding of the potential role of miRNAs in multidrug resistance of the insulin-resistant HCC cells.

\section{Materials and Methods}

\section{Cell culture}

Human hepatocellular carcinoma HepG2 cells were purchased from American Type Culture
Collection (ATCC HB-8065, Rockville, MD, USA) and cultured in Dulbecco's modified eagle medium (DMEM) supplemented with $10 \%$ FBS at $37^{\circ} \mathrm{C}$ with $5 \% \mathrm{CO}_{2}$. IR was induced in HepG2 cells according to the method described previously [7]. Briefly, cells were incubated in serum-free DMEM for $6 \mathrm{hr}$ to synchronization then treated with insulin at a concentration of $0.5 \mu \mathrm{M}$ for $72 \mathrm{hr}$. The resultant cells were named as HepG2/IR cells.

\section{RNA extraction}

Total RNAs from each sample were individually isolated using QIAzol Lysis Reagent and miRNeasy mini kit (Qiagen Inc, Valencia, CA, USA) according to the manufacturers' instructions. This procedure efficiently recovered all types of RNAs, including miRNAs. RNA quantity and quality were measured using a NanoDrop spectrophotometer (ND-1000; Nanodrop Technologies, Wilmington, DE, USA) and RNA integrity was detected by gel electrophoresis.

\section{Affymetrix GeneChip miRNA Array Hybridization}

For analysis with Affymetrix GeneChip miRNA Arrays (Affymetrix, Santa Clara, CA, USA), each sample was prepared with $150 \mathrm{ng}$ of total RNAs. Samples were labeled with the FlashTag Biotin HSR labeling kit (Affymetrix, Santa Clara, CA, USA) according to the manufacturer's instructions. The RNA sample was mixed into the Poly A Tailing master mix, and FlashTagTM Biotin HSR Ligation was performed by adding FlashTag Biotin HSR Ligation Mix to each of the tailed RNA samples. T4 DNA Ligase was added to each sample for labeling reaction. The hybridization cocktail was then added to each labeled sample, the resultant mix of each sample was applied to an array on the Affymetrix GeneChip miRNA 4.0 Arrays, the probe arrays were washed and stained, then scanned and analyzed using the Affymetrix GeneChip Fluidics Station 450. Differentially expressed miRNAs were identified using fold changes as well as P values calculated by the Student's t-test. The thresholds set for up and down regulated miRNAs were a fold change $\geq 2.0$ and $P \leq 0.05$.

\section{Bioinformatics analysis}

Potential target genes of differentially expressed miRNAs were predicted using DIANA-miRPath online software [19], with Micro T threshold set at 0.8 and P-value threshold set at 0.05 . The predicted target genes subsequently underwent Gene Ontology (GO) analysis for functional annotation analysis and Kyoto Encyclopedia of Genes and Genomes (KEGG) database analysis to identify the enriched functions and pathways that might be involved. Fisher's exact and Chi-square tests were used to determine the 
significance of the GO and KEGG terms and pathways. Only the KEGG terms with a P-value $<0.01$ and GO terms with a P-value $<1 \mathrm{E}-10$ were selected.

\section{miRNA-gene network analyses}

To improve the understanding of the associations between the differentially expressed miRNAs and its target genes, miRNA-gene network was constructed according to the regulatory associations between miRNAs and target genes. The associations of the genes and miRNAs were constructed by Cytoscape 3.7.2 software. The output degree was used to measure the regulated effect of miRNAs on genes or effect of genes on miRNAs.

\section{miRNA-GO network analysis}

The miRNA-GO network was constructed according to the significantly expressed miRNAs and the results of the GO analysis. The degree of miRNAs indicated the number of GOs which were regulated by the miRNA in the network. In the same way, a higher degree of GO indicated that more miRNAs involved in the GO category. Similarly, a higher degree of miRNA suggested more GO categories related with certain miRNA.

\section{miRNA-KEGG network analysis}

A miRNA-KEGG network was constructed according to the specifically regulated miRNAs and the KEGG analysis. The degree of miRNAs indicated the number of KEGG pathways which was regulated by the miRNA in the network. In the same way, a higher degree of KEGG indicated that more miRNAs involved in the KEGG pathway.

\section{Quantitative reverse transcription polymerase chain reaction (qRT-PCR)}

To validate the authenticity of miRNA expression detected by microarray assay and the crucial predicted target genes of these miRNAs, 14 differentially expressed miRNAs were analyzed by qRT-PCR, including down-regulated miRNAs such: as hsa-miR134-5p, hsa-miR-5195-3p, hsa-miR-641, hsa-miR-3935 and; up-regulated miRNAs such as hsa-miR-1231 (ID: hsmq-0529), hsa-miR-6726-5p (ID: hsmq-2842), hasmiR-3180 (ID: hsmq-1188), sa-miR-3613-5p (ID: hsmq1112), hsa-miR-6779-5p (ID: hsmq-2840), hsa-miR7111-5p (ID: hsmq-2216), hsa-miR-6870-5p (ID: hsmq-2604), hsa-miR-6813-5p (ID: hsmq-2845), hsamiR-4492 (ID: hsmq-1344) and hsa-miR-4505 (ID: hsmq-1358). For this analysis, the miRNAs were detected using the All-in-One ${ }^{\mathrm{TM}}$ miRNA qRT-PCR Detection Kit (GeneCopoeia, Rockville, MD, USA) according to the manufacturer's instructions. Item ID of each primer is showed before. qPCR was performed using SYBR-Green (Thermo Fisher Scientific, Inc.) reagent according to the manufacturer's instructions in the ABI 7300 real-time qPCR system (Thermo Fisher Scientific, Waltham, MA, USA). The relative abundance of each miRNA was calculated by the comparative $\mathrm{Ct}$ method (2- $\Delta \Delta \mathrm{Ct})$, and the results were assessed by using a t-test.

\section{Statistical analysis of $q R T-P C R$ result}

All quantitative data were presented as means \pm standard deviations. Comparisons between HepG2/ IR and it parental cells were performed using the Student's t-test. P-values $<0.05$ were considered statistically significant. Statistical values were calculated using SPSS software, version 20.0 (IBM Corp, Armonk, NY, USA).

\section{Results}

\section{miRNA microarray analysis}

To identify the differentially expressed miRNAs between HepG2/IR cells and its parental cells, both cell lines were subjected to the Affymetrix microRNA 4.0 array in triplicate. The results revealed that, of all the 2006 miRNAs screened, 32 miRNAs were dysregulated in HepG2/IR cells compared with its normal control (Table 1) under the condition of 'fold change $>2$ and $P<0.05^{\prime}$. Among these 32 miRNAs, 27 were up-regulated and 5 were down-regulated. An unsupervised 2D-cluster analysis was applied for these two cell lines (Fig. 1) Volcano plot of significantly expressed miRNAs between HepG2/IR and HepG2 cells were showed in Fig. 2 and the 32 of them were highlighted.

\section{Target genes and bioinformatics analysis}

A total of 5,406 genes were predicted as target genes of the identified 32 differentially expressed miRNAs (Table S1). To better understand the potential implications of these dysregulated miRNAs, the target genes were subjected to GO analysis to evaluate their potential functions using DIANA-miRPath v3.0 online software (http:/ / snf-515788.vm.okeanos.grnet. $\mathrm{gr} /$ ). In the present study, the top GO terms of the target genes of the dysregulated miRNAs were regulation of cellular nitrogen compound metabolic process, biosynthetic process, cellular protein modification process (BP); cellular component, protein complex, cytosol (CC); ion binding, transcription coactivator activity, molecular function, nucleic acid binding transcription factor activity (MF) (Fig. 3, Table S2).

The target genes were also subjected to KEGG pathway enrichment analysis using DIANA-miRPath v3.0 online software (http://snf-515788.vm.okeanos. grnet.gr/) to determine the canonical pathways controlled by the identified miRNAs. Other types of 
O-glycan biosynthesis, proteoglycans in cancer, adrenergic signaling in cardiomyocytes, estrogen signaling pathway, cell adhesion molecules, glycosphingolipid biosynthesis, glycosaminoglycan biosynthesis, and ErbB signaling pathway were the most active pathways that the target genes of the differentially expressed miRNAs may be involved. (Fig. 4, Table S3).

\section{miRNA-gene-network analyses}

A miRNA-gene network was constructed according to the results of the GO and KEGG pathway analyses. The core miRNAs of the interaction network include miR-6870-5p, miR-7111-5p, miR-4505, miR4492 and miR-641 (Table 2). The network also revealed that Kinase suppressor of Ras 2 (KSR2), Ras/ Rap GTPase-activating protein SynGAP (SYNGAP1), Glutamate receptor ionotropic (GRIN2B), G proteinactivated inward rectifier potassium channel 2 (KCNJ6), and Complexin-2 (CPLX2) were the most crucial target genes (Table 3, Table S1). The associations of miRNAs with genes were shown in Fig. 5.

Table 1. Differentially expressed microRNAs (miRNAs) between HepG2/IR cells and its parental control $(p<0.05$, Fold change $>2)$

\begin{tabular}{llll}
\hline miRNA & $\mathrm{p}$-value & Fold Change & Type of regulation \\
\hline hsa-miR-3613-5p & 0.0242590 & 6.28 & $\mathrm{Up}$ \\
hsa-miR-6813-5p & 0.0123839 & 6.12 & $\mathrm{Up}$ \\
hsa-miR-6132 & 0.0099436 & 4.05 & $\mathrm{Up}$ \\
hsa-miR-4492 & 0.0160402 & 3.59 & $\mathrm{Up}$ \\
hsa-miR-3937 & 0.0468370 & 3.36 & $\mathrm{Up}$ \\
hsa-miR-7112-5p & 0.0283560 & 3.31 & $\mathrm{Up}$ \\
hsa-miR-6795-5p & 0.0137803 & 3.28 & $\mathrm{Up}$ \\
hsa-miR-6726-5p & 0.0052162 & 3.11 & $\mathrm{Up}$ \\
hsa-miR-6848-5p & 0.0044207 & 3.07 & $\mathrm{Up}$ \\
hsa-miR-6870-5p & 0.0001978 & 3.01 & $\mathrm{Up}$ \\
hsa-miR-7111-5p & 0.0247380 & 2.94 & $\mathrm{Up}$ \\
hsa-miR-6780b-5p & 0.0398410 & 2.90 & $\mathrm{Up}$ \\
hsa-miR-6782-5p & 0.0193971 & 2.82 & $\mathrm{Up}$ \\
hsa-miR-3180 & 0.0482000 & 2.73 & $\mathrm{Up}$ \\
hsa-miR-4467 & 0.0296810 & 2.72 & $\mathrm{Up}$ \\
hsa-miR-6805-5p & 0.0174751 & 2.66 & $\mathrm{Up}$ \\
hsa-miR-6846-5p & 0.0231760 & 2.54 & $\mathrm{Up}$ \\
hsa-miR-4706 & 0.0456770 & 2.48 & $\mathrm{Up}$ \\
hsa-miR-4505 & 0.0307830 & 2.38 & $\mathrm{Up}$ \\
hsa-miR-8072 & 0.0176760 & 2.37 & $\mathrm{Up}$ \\
hsa-miR-6085 & 0.0333000 & 2.28 & $\mathrm{Up}$ \\
hsa-miR-4649-5p & 0.0200810 & 2.25 & $\mathrm{Up}$ \\
hsa-miR-6757-5p & 0.0040993 & 2.23 & $\mathrm{Up}$ \\
hsa-miR-6779-5p & 0.0167355 & 2.18 & $\mathrm{Up}$ \\
hsa-miR-3621 & 0.0468600 & 2.13 & $\mathrm{Up}$ \\
hsa-miR-1231 & 0.033018 & 2.08 & $\mathrm{Up}$ \\
hsa-miR-4508 & 0.0143212 & 2.04 & $\mathrm{Up}$ \\
hsa-miR-641 & 0.027156 & -2.23 & $\mathrm{Down}$ \\
hsa-miR-4793-5p & 0.0133985 & -2.27 & $\mathrm{Down}$ \\
hsa-miR-134-5p & 0.040759 & -2.95 & $\mathrm{Down}$ \\
hsa-miR-5195-3p & 0.00055787 & -3.30 & $\mathrm{Down}$ \\
hsa-miR-3935 & 0.030403 & -3.55 & $\mathrm{Down}$ \\
\hline
\end{tabular}

Table 2. Crucial microRNAs (miRNAs) in the miRNA-target network (degree $>3$ )

\begin{tabular}{ll}
\hline miRNA & Degree \\
\hline hsa-miR-6870-5p & 87 \\
hsa-miR-7111-5p & 67 \\
hsa-miR-4505 & 66 \\
hsa-miR-4492 & 57 \\
hsa-miR-641 & 52 \\
hsa-miR-6780b-5p & 50 \\
hsa-miR-5195-3p & 48 \\
hsa-miR-6846-5p & 46 \\
hsa-miR-6848-5p & 45 \\
hsa-miR-6779-5p & 41 \\
hsa-miR-6795-5p & 27 \\
hsa-miR-6132 & 26 \\
hsa-miR-134-5p & 22 \\
hsa-miR-3613-5p & 19 \\
hsa-miR-1231 & 18 \\
hsa-miR-4793-5p & 17 \\
hsa-miR-6085 & 17 \\
hsa-miR-6813-5p & 16 \\
hsa-miR-6782-5p & 14 \\
hsa-miR-3935 & 13 \\
hsa-miR-6757-5p & 13 \\
hsa-miR-6726-5p & 8 \\
hsa-miR-6805-5p & 8 \\
hsa-miR-3180 & 4 \\
\hline
\end{tabular}

\section{miRNA-GO network analysis}

The miRNA-GO network analysis was favourable for determining regulatory associations between the key miRNAs and hub GO. In this network, miR-4492, miR-641 and miR-6779-5p, which contributed more than the other specifically expressed miRNAs exhibit 114, 114 and 113 GO functions, respectively (Table 4 , Table S4). The most significantly regulated functional clusters of total categories were cellular nitrogen compound metabolic process, biosynthetic process, biological process and catabolic process (Table 5). The significantly complicated categories associations of miRNAs with degree more than 25 were shown in Fig. 6.

\section{miRNA-KEGG network analysis}

The network analysis was also employed to determine regulatory associations between the key miRNAs and hub KEGG pathways. In this network, miR-4492, miR-5193-3p and miR-641, which contributed more than other specifically expressed miRNAs exhibited 45, 44 and 44 KEGG pathways respectively (Table 6, Table S5). The most significantly KEGG pathway were MAPK signaling pathway, Ras signaling pathway, endocytosis and Rap1 signaling pathway, which involved in cell proliferation, growth, and differentiation (Table 7). The significantly complicated pathway associations of miRNAs with degree more than 30 are shown in Fig. 7. 


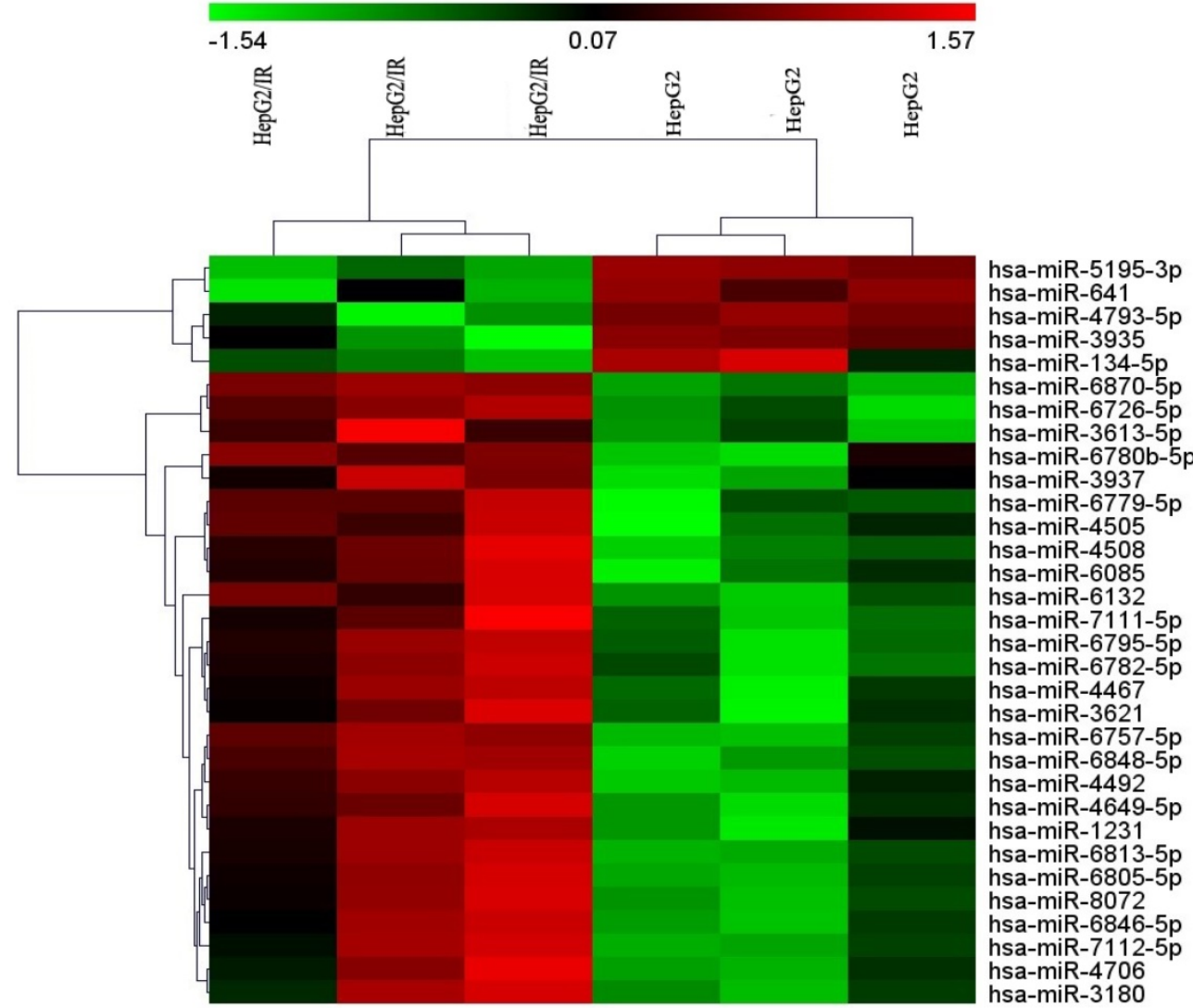

Figure 1. Heatmap of the microarray hybridization result showed the differentially expressed miRNAs between HepG2/IR cells and its parental cells (HepG2 cells). Total RNA was extracted from both of two cell lines, miRNAs microarrays were performed as described in Materials and Methods, both cell lines were tested in triplicate.

\section{Validation of miRNA array data}

Based on their expression levels and fold changes, 4 downregulated miRNAs (miR-134-5p, miR-5195-3p, miR-641, miR-3935) and 10 upregulated miRNAs (miR-1231, miR-6726-5p, miR-3613-5p, miR6779-5p, miR-7111-5p, miR-6870-5p, miR-6813-5p, miR-4492, miR-4505, miR-3180) were selected for validation by qRT-PCR. The results revealed a significant difference of the expression level of miR-4492, miR-3180, miR-134-5p, miR-5195-3p, miR641 between the IR cells and control cells, which was in a manner consistent with the data obtained from microarray analysis $(\mathrm{P}<0.05)$ (Fig. 8). GO enrichment analysis revealed that these validated miRNAs were significantly enriched in biosynthetic process, cellular protein modification process, gene expression, small molecule metabolic process, catabolic process, cellular component assembly, post-translational protein modification (Table S4). The pathways were also significantly enriched in the KEGG analysis, and the most involved were cancer-related pathways, including the: Ras signaling pathway, Hippo signaling pathway, ErbB signaling pathway, other types of O-glycan biosynthesis, proteoglycans in cancer and cell adhesion molecules (CAMs) (Table S5).
Table 3. Crucial and hub target genes in the miRNA-target network (degree $>4$ )

\begin{tabular}{|c|c|c|}
\hline Crucial Target Gene & Description & Degree \\
\hline CPLX2 & Complexin-2 & 8 \\
\hline GRIN2B & Glutamate receptor ionotropic, NMDA 2B & 8 \\
\hline KCNJ6 & $\begin{array}{l}\text { G protein-activated inward rectifier } \\
\text { potassium channel } 2\end{array}$ & 8 \\
\hline KSR2 & Kinase suppressor of Ras 2 & 8 \\
\hline SYNGAP1 & $\begin{array}{l}\text { Ras/Rap GTPase-activating protein } \\
\text { SynGAP }\end{array}$ & 8 \\
\hline THRA & Thyroid hormone receptor alpha & 8 \\
\hline AGAP2 & $\begin{array}{l}\text { Arf-GAP with GTPase, ANK repeat and } \\
\text { PH domain-containing protein } 2\end{array}$ & 7 \\
\hline PTCH1 & Protein patched homolog 1 & 7 \\
\hline SEMA4G & Semaphorin-4G & 7 \\
\hline VAMP2 & Vesicle-associated membrane protein 2 & 7 \\
\hline CDK6 & Cyclin-dependent kinase 6 & 6 \\
\hline CRTC2 & $\begin{array}{l}\text { CREB-regulated transcription coactivator } \\
2\end{array}$ & 6 \\
\hline PAK6 & Serine/threonine-protein kinase PAK 6 & 6 \\
\hline PRKCA & Protein kinase $C$ alpha type & 6 \\
\hline ATP2B2 & $\begin{array}{l}\text { Plasma membrane calcium-transporting } \\
\text { ATPase } 2\end{array}$ & 5 \\
\hline CACNB3 & $\begin{array}{l}\text { Voltage-dependent L-type calcium } \\
\text { channel subunit beta-3 }\end{array}$ & 5 \\
\hline FLNB & Filamin-B & 5 \\
\hline PPP1R12B & $\begin{array}{l}\text { Protein phosphatase } 1 \text { regulatory subunit } \\
\text { 12B }\end{array}$ & 5 \\
\hline SLC8A1 & Sodium/calcium exchanger 1 & 5 \\
\hline VPS37D & $\begin{array}{l}\text { Vacuolar protein sorting-associated } \\
\text { protein 37D }\end{array}$ & 5 \\
\hline
\end{tabular}




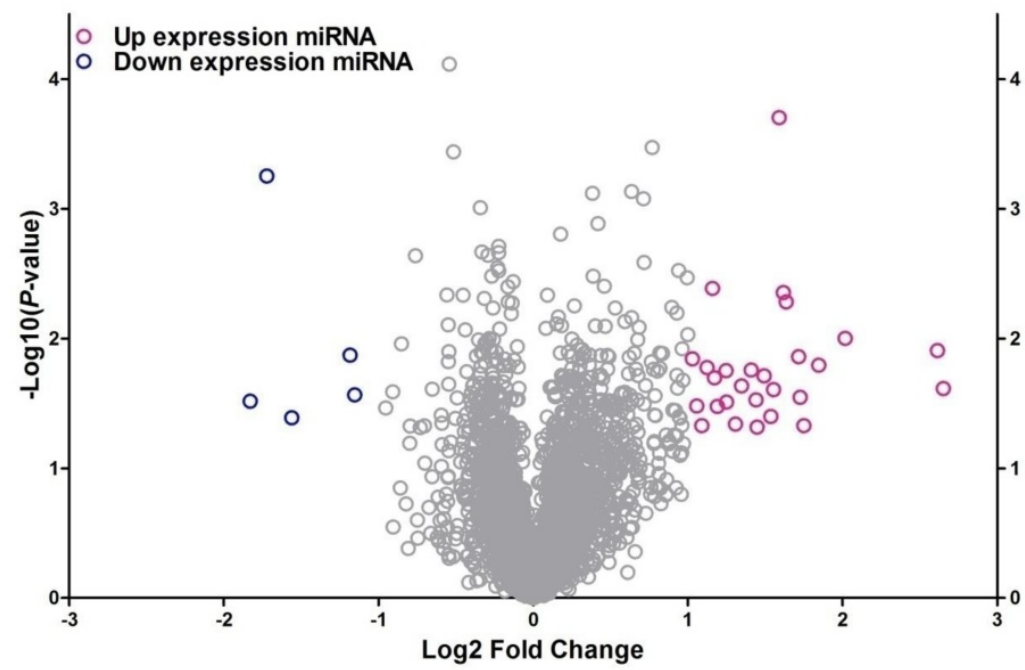

Figure 2: Volcano plot of differentially expressed miRNA between HepG2/IR and HepG2 cells. The x-axis shows the Log2 fold-change in miRNA expression and $y$-axis shows the -Log10 of the p-value from HepG2/IR versus HepG2 cells. Labelled miRNAs have Log2 fold change greater than 1SD from mean.

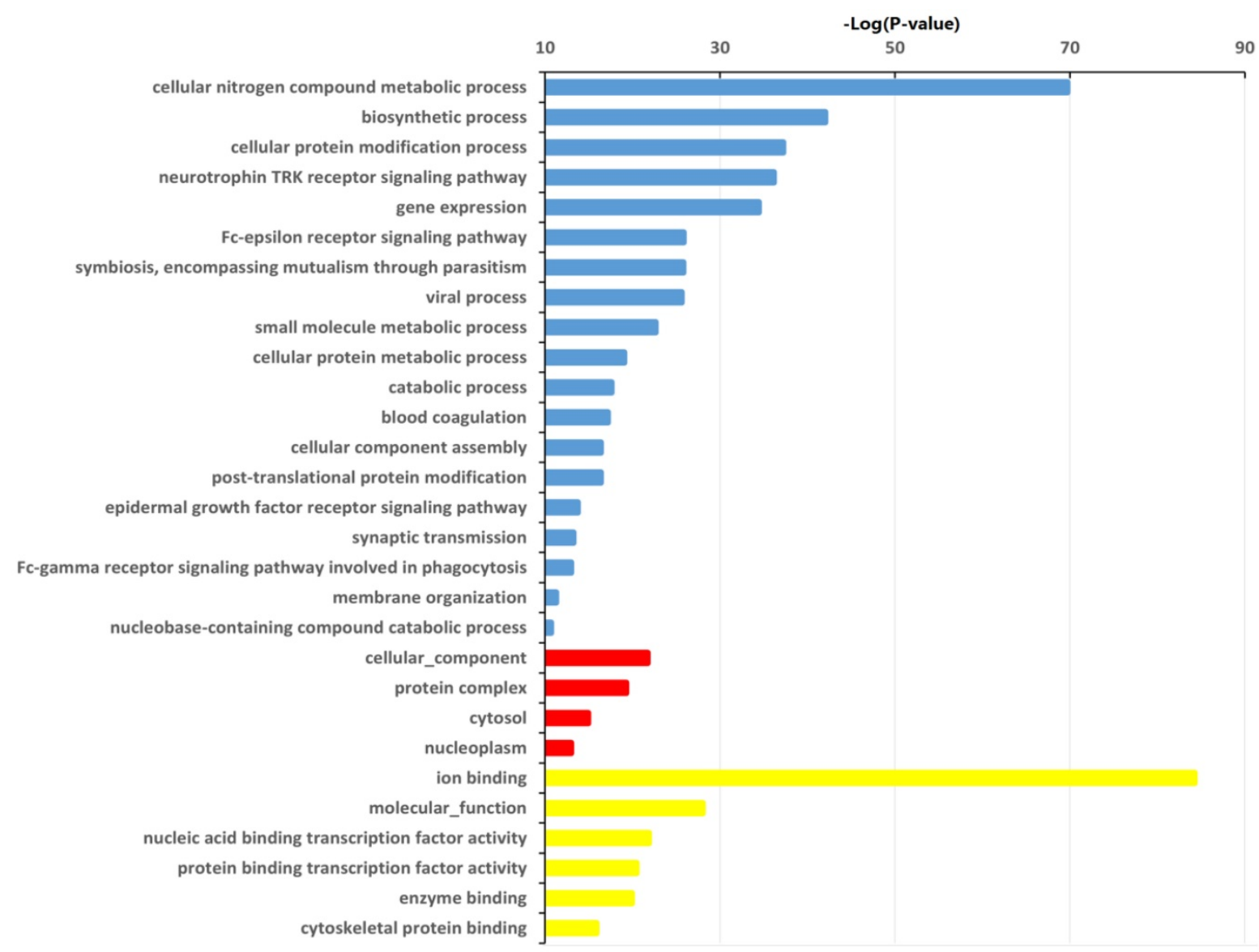

Figure 3. Significantly changed GO categories of predicted target genes of deregulated miRNAs between HepG2/IR and HepG2 cells. The $y$-axis shows GO category and the $x$-axis shows - $\lg P$. The larger $-\lg P$ indicated a smaller $P$ value. $-\lg P$ represent the negative logarithm of the $P$ value. Blue bars indicate biological process (BP), red bars indicate cellular component (CC) and yellow bars indicate molecular function (MF).

\section{Discussion}

As the main anabolic hormone of the body, insulin regulates the metabolism of nutrients and promotes absorption of glucose from the blood into the cells. IR is the status when cells in the body, especially cells in the liver, fat and muscles don't respond well to insulin, and correspondingly the uptake and utilization of glucose decreases. At the whole organism level, IR will cause an impaired effect of insulin with lowering blood glucose, which may develop T2DM. To compensate for low blood glucose, 
the pancreas compensatory secretes excessive insulin to maintain blood sugar stability and to help glucose entering the cells. This excessive secretion leads to hyperinsulinemia in the body, which in turn activates hepatic lipogenesis and increased secretion of VLDL (hyperlipidemia) [20].

Table 4. Crucial miRNAs in the miRNA-GO network (degree $>10$ )

\begin{tabular}{ll}
\hline miRNA & Degree \\
\hline hsa-miR-4492 & 114 \\
hsa-miR-641 & 114 \\
hsa-miR-6779-5p & 113 \\
hsa-miR-6870-5p & 113 \\
hsa-miR-7111-5p & 110 \\
hsa-miR-3613-5p & 109 \\
hsa-miR-6780b-5p & 108 \\
hsa-miR-4505 & 106 \\
hsa-miR-6846-5p & 105 \\
hsa-miR-6795-5p & 104 \\
hsa-miR-134-5p & 101 \\
hsa-miR-4793-5p & 101 \\
hsa-miR-6848-5p & 101 \\
hsa-miR-5195-3p & 100 \\
hsa-miR-1231 & 97 \\
hsa-miR-6132 & 97 \\
hsa-miR-6757-5p & 97 \\
hsa-miR-6813-5p & 96 \\
hsa-miR-6782-5p & 92 \\
hsa-miR-6085 & 91 \\
hsa-miR-3935 & 81 \\
hsa-miR-3937 & 74 \\
hsa-miR-6805-5p & 67 \\
hsa-miR-6726-5p & 63 \\
hsa-miR-3180 & 59 \\
hsa-miR-4649-5p & 58 \\
hsa-miR-4508 & 33 \\
hsa-miR-4706 & 33 \\
hsa-miR-7112-5p & 33 \\
hsa-miR-4467 & 29 \\
hsa-miR-3621 & 20 \\
hsa-miR-8072 & 16 \\
\hline
\end{tabular}

Nonalcoholic fatty liver disease (NAFLD), as one of the most common liver disorders worldwide [21], had already been reported to have tight association with IR. Although NAFLD includes a disease spectrum, ranging from simple steatosis, liver cirrhosis to hepatocellular carcinoma (HCC) etc. [22,23] and the overall occurrence from NAFLD to HCC remains a rare complication [24,25]. IR had been reported to be associated with increased risk of several cancers, including HCC [26,27]. It had not only been related to tumorigenesis, but also been correlated to the poor prognosis of cancer patients [3-5]. Our previous studies also proved that IR could increase the invasiveness and MDR in the HCC cell line [6-9]. However, the molecular mechanism of therapeutic resistance caused by IR still remains unclear.

Our present study demonstrated that 27
miRNAs were consistently upregulated and 5 miRNAs were downregulated in the HepG2/IR cells upon triplicate tests. qRT-PCR was employed to verify the expression data of 14 differentially expressed miRNAs, which showed that the expression of 5 miRNAs were in consistent with the microarray data. The outcome of qRT-PCR validated that our result of microarray screening was reliable. Among these validated miRNAs, some had already been reported to be correlated with cancer. For instance, miR-134-5p had been reported as a tumor suppressing miRNA involving in several cancers. The anti-tumor mechanisms of miR-134-5p as the potential target of various lncRNAs which function as oncogene and it can also inhibit the expression of tumor suppressor genes by directly binding to the $3^{\prime} U T R$ region. It was confirmed that impairing the expression of miR134-5p can promote cell proliferation, inhibit cell apoptosis, accelerate cell migration, invasion, and induce EMT and moreover contribute to multidrug resistance such as cisplatin (targeting MBTD1), 5-FU (targeting KRAS) and paclitaxel (targeting TAB1) in several carcinoma cells including non-small-cell lung, gastric, nasopharyngeal, ovarian cancers and osteosarcoma [32-35,55,56]. Another microRNA reported was miR-641 which has been proved as a tumor suppressor; inhibiting proliferation, migration and invasion, as well as inducing apoptosis in lung cancer, cervical cancer and glioblastoma [36-38]. It was also confirmed that miR-641 contributes to erlotinib resistance in non-small-cell lung cancer by targeting NF1 and regulating ERK signaling [39]. However, little was known about the role of miR-641 in HCC. The only report about miR-641 and drug metabolism is that it acts as a direct post-transcriptional regulatory factor of CYP3A4, which is a member of the cytochrome P450 superfamily. As we all know, the cytochrome $\mathrm{P} 450$ proteins are monooxygenases in the hepatocytes that catalyze many reactions involved in drug metabolism, the enzyme also metabolizes some steroids and carcinogens as well [40]. Until now, the functions especially in tumorigenesis and drug resistance of other validated miRNAs such as miR-3180, miR-6726-5p, miR-7111-5p etc. have remained unclear. Our previous study has reported that insulin resistant HCC cells obtain ability of multidrug resistance such as cisplatin, 5-FU, vincristine, mitomycin [7]. In accordance with the researches mentioned above, miR-134-5p and miR-641 were down-regulated in insulin resistant HCC cells, thus suggesting that these known or unknown miRNAs might play a critical role in multidrug resistance of insulin resistant HCC cells especially miR-134-5p and miR-641. Further functional experiments will be conducted in our following study. 
Table 5. Crucial GO category in the microRNA-GO network (degree $>20$ )

\begin{tabular}{|c|c|c|c|c|}
\hline \multicolumn{2}{|c|}{ GO ID } & \multirow{2}{*}{$\begin{array}{l}\text { GO (name) } \\
\text { cellular nitrogen compound metabolic process }\end{array}$} & \multirow{2}{*}{$\begin{array}{l}\text { Degree } \\
32\end{array}$} & \multirow{2}{*}{$\begin{array}{l}\text { P value } \\
1.93 \mathrm{E}-70\end{array}$} \\
\hline $\mathrm{BP}$ & GO:0034641 & & & \\
\hline $\mathrm{BP}$ & GO:0009058 & biosynthetic process & 32 & 8.87E-43 \\
\hline $\mathrm{BP}$ & GO:0008150 & biological_process & 32 & 2.79E-06 \\
\hline $\mathrm{BP}$ & GO:0009056 & catabolic process & 31 & $2.45 \mathrm{E}-18$ \\
\hline $\mathrm{BP}$ & GO:0022607 & cellular component assembly & 31 & $4.15 \mathrm{E}-17$ \\
\hline $\mathrm{BP}$ & GO:0006351 & transcription, DNA-templated & 31 & 0.0449519 \\
\hline $\mathrm{BP}$ & GO:0006464 & cellular protein modification process & 30 & $5.95 \mathrm{E}-38$ \\
\hline $\mathrm{BP}$ & GO:0044281 & small molecule metabolic process & 30 & $2.21 \mathrm{E}-23$ \\
\hline $\mathrm{BP}$ & GO:0006950 & response to stress & 30 & 3.49E-09 \\
\hline $\mathrm{BP}$ & GO:0034655 & nucleobase-containing compound catabolic process & 29 & $1.98 \mathrm{E}-11$ \\
\hline $\mathrm{BP}$ & GO:0065003 & macromolecular complex assembly & 29 & 1.19E-10 \\
\hline $\mathrm{BP}$ & GO:0006461 & protein complex assembly & 29 & $3.48 \mathrm{E}-07$ \\
\hline $\mathrm{BP}$ & GO:0016192 & vesicle-mediated transport & 29 & 0.0433165 \\
\hline $\mathrm{BP}$ & GO:0002376 & immune system process & 28 & $2.04 \mathrm{E}-06$ \\
\hline $\mathrm{BP}$ & GO:0008219 & cell death & 28 & 2.97E-06 \\
\hline $\mathrm{BP}$ & GO:0006259 & DNA metabolic process & 28 & 0.0073611 \\
\hline $\mathrm{BP}$ & GO:0010467 & gene expression & 27 & $3.60 \mathrm{E}-35$ \\
\hline $\mathrm{BP}$ & GO:0044403 & symbiosis, encompassing mutualism through parasitism & 27 & $1.57 \mathrm{E}-26$ \\
\hline $\mathrm{BP}$ & GO:0044267 & cellular protein metabolic process & 27 & 8.96E-20 \\
\hline $\mathrm{BP}$ & GO:0048011 & neurotrophin TRK receptor signaling pathway & 26 & $6.94 \mathrm{E}-37$ \\
\hline $\mathrm{BP}$ & GO:0016032 & viral process & 26 & $2.41 \mathrm{E}-26$ \\
\hline $\mathrm{BP}$ & GO:0043687 & post-translational protein modification & 26 & 4.23E-17 \\
\hline $\mathrm{BP}$ & GO:0061024 & membrane organization & 26 & $5.18 \mathrm{E}-12$ \\
\hline $\mathrm{BP}$ & GO:0007267 & cell-cell signaling & 26 & 1.13E-09 \\
\hline $\mathrm{BP}$ & GO:0051056 & regulation of small GTPase mediated signal transduction & 26 & 1.07E-05 \\
\hline $\mathrm{BP}$ & GO:0006367 & transcription initiation from RNA polymerase II promoter & 26 & 5.57E-05 \\
\hline $\mathrm{BP}$ & GO:0042592 & homeostatic process & 26 & 0.0079643 \\
\hline $\mathrm{BP}$ & GO:0030198 & extracellular matrix organization & 26 & 0.0361203 \\
\hline $\mathrm{BP}$ & GO:0007596 & blood coagulation & 25 & $6.77 \mathrm{E}-18$ \\
\hline $\mathrm{BP}$ & GO:0007268 & synaptic transmission & 25 & $5.55 \mathrm{E}-14$ \\
\hline $\mathrm{BP}$ & GO:0016070 & RNA metabolic process & 25 & $3.88 \mathrm{E}-06$ \\
\hline $\mathrm{BP}$ & GO:0045087 & innate immune response & 25 & 0.0002734 \\
\hline $\mathrm{BP}$ & GO:0048870 & cell motility & 25 & 0.000421 \\
\hline $\mathrm{BP}$ & GO:0007010 & cytoskeleton organization & 25 & 0.0095913 \\
\hline $\mathrm{BP}$ & GO:0016071 & mRNA metabolic process & 24 & 3.49E-09 \\
\hline $\mathrm{BP}$ & GO:0007411 & axon guidance & 24 & 0.0001162 \\
\hline $\mathrm{BP}$ & GO:0097190 & apoptotic signaling pathway & 24 & 0.0084989 \\
\hline $\mathrm{BP}$ & GO:0007399 & nervous system development & 24 & 0.0092641 \\
\hline $\mathrm{BP}$ & GO:0006790 & sulfur compound metabolic process & 24 & 0.0392062 \\
\hline $\mathrm{BP}$ & GO:0038095 & Fc-epsilon receptor signaling pathway & 23 & $1.46 \mathrm{E}-26$ \\
\hline $\mathrm{BP}$ & GO:0007173 & epidermal growth factor receptor signaling pathway & 23 & $1.72 \mathrm{E}-14$ \\
\hline $\mathrm{BP}$ & GO:0030203 & glycosaminoglycan metabolic process & 23 & $2.21 \mathrm{E}-10$ \\
\hline $\mathrm{BP}$ & GO:0000278 & mitotic cell cycle & 23 & 2.73E-08 \\
\hline $\mathrm{BP}$ & GO:0030168 & platelet activation & 23 & 3.73E-07 \\
\hline $\mathrm{BP}$ & GO:0008286 & insulin receptor signaling pathway & 23 & 2.61E-06 \\
\hline $\mathrm{BP}$ & GO:0019221 & cytokine-mediated signaling pathway & 23 & 0.0327518 \\
\hline $\mathrm{BP}$ & GO:0008543 & fibroblast growth factor receptor signaling pathway & 22 & 3.49E-09 \\
\hline $\mathrm{BP}$ & GO:0034142 & toll-like receptor 4 signaling pathway & 22 & 2.23E-08 \\
\hline $\mathrm{BP}$ & GO:0034330 & cell junction organization & 22 & 3.30E-06 \\
\hline $\mathrm{BP}$ & GO:0006325 & chromatin organization & 22 & 0.0046663 \\
\hline $\mathrm{BP}$ & GO:0022617 & extracellular matrix disassembly & 22 & 0.0138599 \\
\hline $\mathrm{BP}$ & GO:0006091 & generation of precursor metabolites and energy & 22 & 0.0460089 \\
\hline $\mathrm{BP}$ & GO:0038096 & Fc-gamma receptor signaling pathway involved in phagocytosis & 21 & $1.01 \mathrm{E}-13$ \\
\hline $\mathrm{BP}$ & GO:0034162 & toll-like receptor 9 signaling pathway & 21 & $1.01 \mathrm{E}-09$ \\
\hline $\mathrm{BP}$ & GO:0002224 & toll-like receptor signaling pathway & 21 & $6.22 \mathrm{E}-07$ \\
\hline $\mathrm{BP}$ & GO:0048015 & phosphatidylinositol-mediated signaling & 21 & $1.05 \mathrm{E}-06$ \\
\hline $\mathrm{BP}$ & GO:0006928 & cellular component movement & 21 & $9.77 \mathrm{E}-05$ \\
\hline $\mathrm{BP}$ & GO:0006644 & phospholipid metabolic process & 21 & 0.0001544 \\
\hline $\mathrm{BP}$ & GO:0000086 & $\mathrm{G} 2 / \mathrm{M}$ transition of mitotic cell cycle & 21 & 0.0024742 \\
\hline $\mathrm{CC}$ & GO:0005575 & cellular_component & 32 & $1.93 \mathrm{E}-22$ \\
\hline $\mathrm{CC}$ & GO:0043234 & protein complex & 30 & 5.11E-20 \\
\hline $\mathrm{CC}$ & GO:0005829 & cytosol & 30 & $1.17 \mathrm{E}-15$ \\
\hline $\mathrm{CC}$ & GO:0005654 & nucleoplasm & 30 & $1.03 \mathrm{E}-13$ \\
\hline $\mathrm{CC}$ & GO:0005815 & microtubule organizing center & 26 & 0.0031329 \\
\hline MF & GO:0043167 & ion binding & 32 & $5.38 \mathrm{E}-85$ \\
\hline MF & GO:0003674 & molecular_function & 32 & $9.58 \mathrm{E}-29$ \\
\hline
\end{tabular}




\begin{tabular}{|c|c|c|c|c|}
\hline GO ID & & GO (name) & Degree & $P$ value \\
\hline $\mathrm{MF}$ & GO:0001071 & nucleic acid binding transcription factor activity & 30 & $1.33 \mathrm{E}-22$ \\
\hline MF & GO:0000988 & protein binding transcription factor activity & 27 & 3.47E-21 \\
\hline MF & GO:0019899 & enzyme binding & 27 & $1.21 \mathrm{E}-20$ \\
\hline MF & GO:0008092 & cytoskeletal protein binding & 28 & $1.25 \mathrm{E}-16$ \\
\hline MF & GO:0030234 & enzyme regulator activity & 29 & 2.03E-10 \\
\hline MF & GO:0022857 & transmembrane transporter activity & 28 & $9.52 \mathrm{E}-05$ \\
\hline MF & GO:0030674 & protein binding, bridging & 23 & 0.0343038 \\
\hline
\end{tabular}

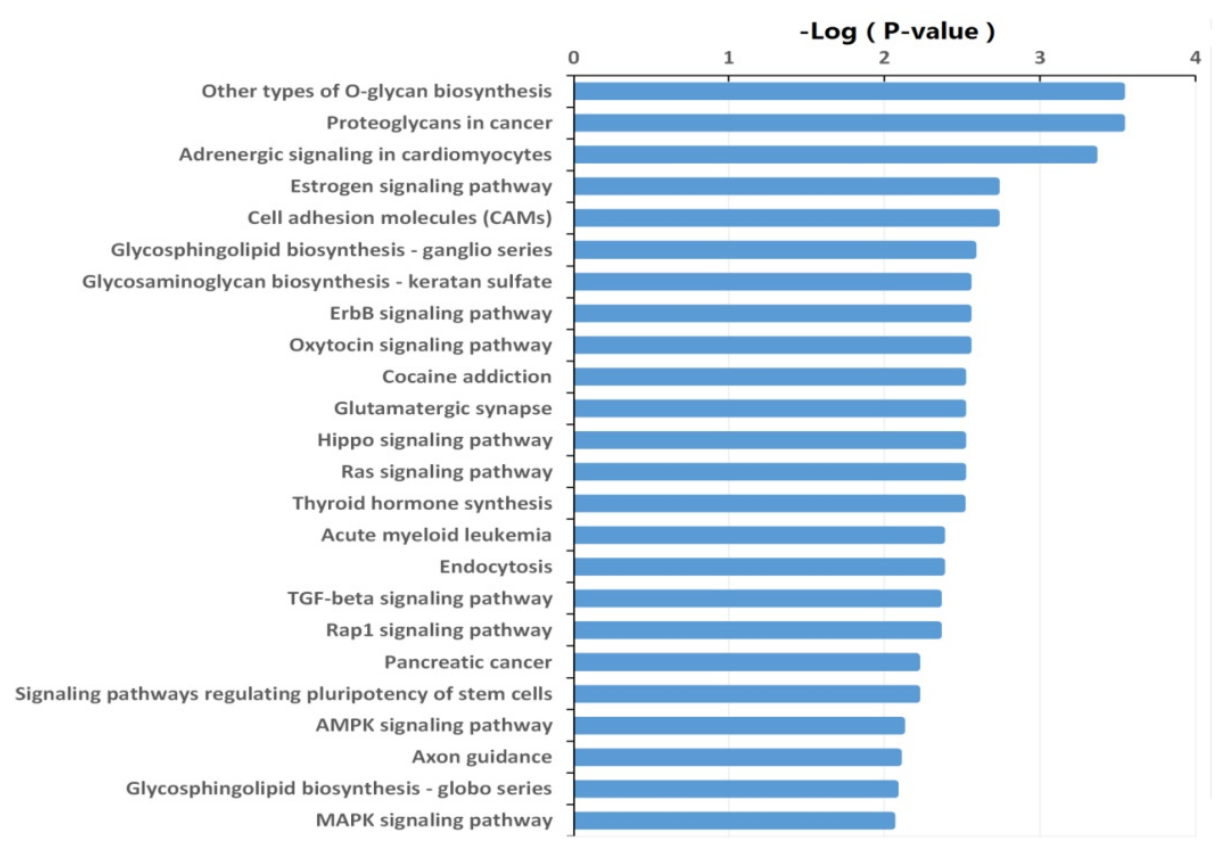

Figure 4. Significantly changed KEGG pathways of predicted target genes of deregulated miRNAs between HepG2/IR and HepG2 cells. The $y$-axis shows KEGG category and the $x$-axis shows - IgP. The larger -lgP indicated a smaller $P$ value. - $\lg P$ represent the negative logarithm of the $P$ value.

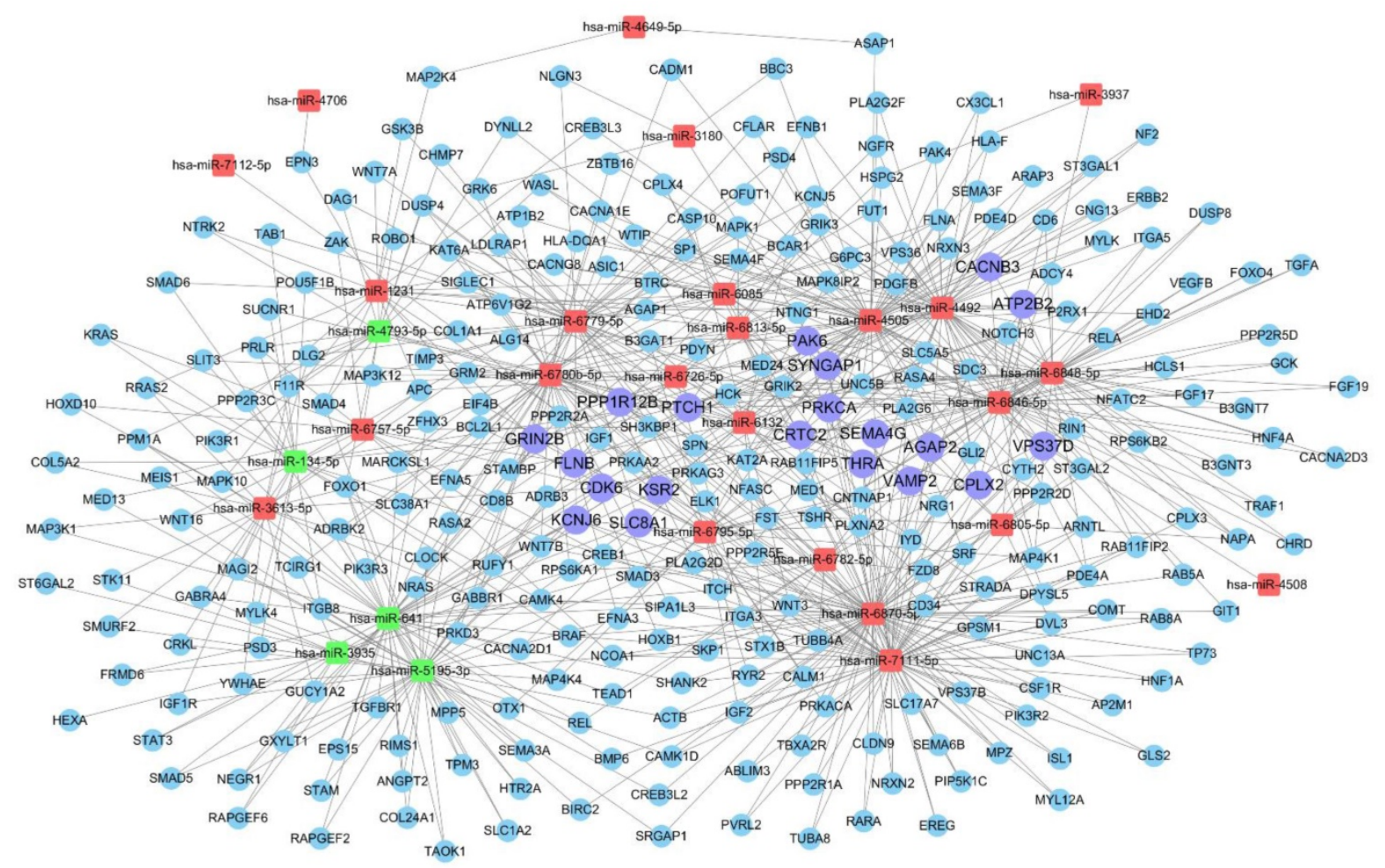

Figure 5. miRNA-gene network showing the interactions between key miRNAs and the predicted hub genes. The square nodes represent miRNAs (red nodes denote up-regulated miRNAs, green nodes denote down-regulated miRNAs), circular nodes represent hub target genes (purple nodes denote crucial and hub target genes with degree $>4$ ). 


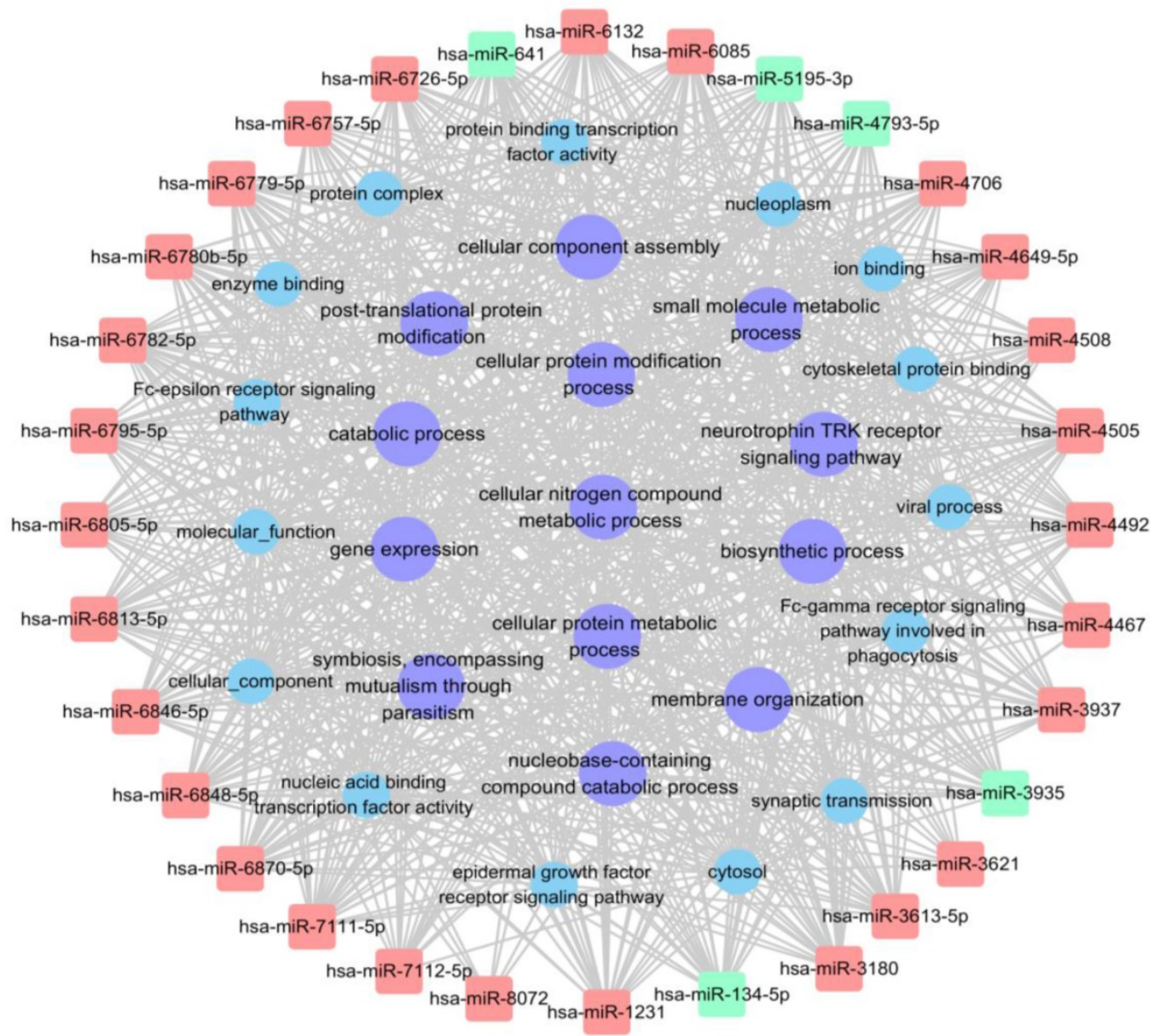

Figure 6. miRNA-GO network showing the interactions between key miRNAs and the hub GO. The square nodes represent miRNAs (red nodes denote up-regulated miRNAs, green nodes denote down-regulated miRNAs), circular nodes represent hub GO with degree $>25$ (purple nodes denote tumor related hub GO).

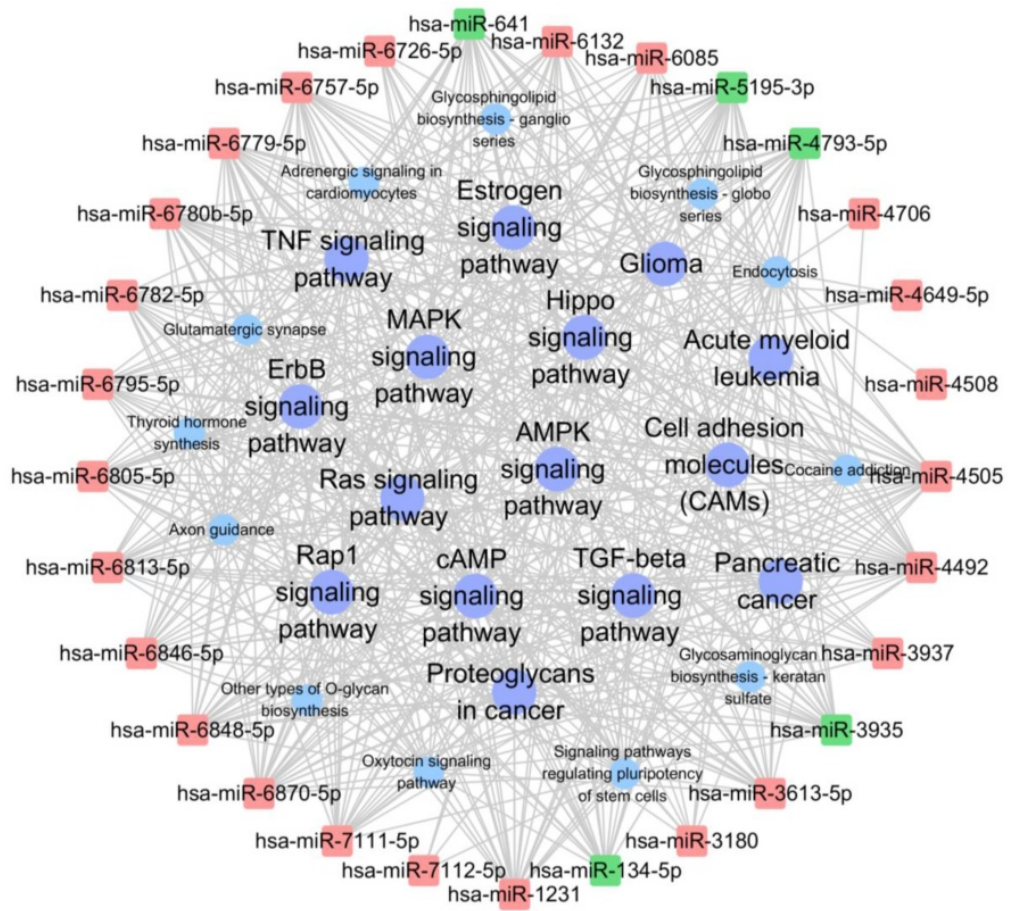

Figure 7. miRNA-KEGG network showing the interactions between key miRNAs and the hub KEGG pathway. The square nodes represent miRNAs (red nodes denote up-regulated miRNAs, green nodes denote down-regulated miRNAs), circular nodes represent hub KEGG pathway with degree>30 (purple nodes denote tumor related hub KEGG). 
Table 6. Crucial miRNAs in the miRNA-KEGG network (degree $>10$ )

\begin{tabular}{ll}
\hline miRNA & Degree \\
\hline hsa-miR-4492 & 45 \\
hsa-miR-5195-3p & 44 \\
hsa-miR-641 & 44 \\
hsa-miR-4505 & 43 \\
hsa-miR-6870-5p & 43 \\
hsa-miR-6848-5p & 42 \\
hsa-miR-6779-5p & 41 \\
hsa-miR-134-5p & 39 \\
hsa-miR-6780b-5p & 39 \\
hsa-miR-6795-5p & 39 \\
hsa-miR-7111-5p & 39 \\
hsa-miR-1231 & 38 \\
hsa-miR-6846-5p & 38 \\
hsa-miR-4793-5p & 37 \\
hsa-miR-6757-5p & 37 \\
hsa-miR-3613-5p & 34 \\
hsa-miR-3935 & 32 \\
hsa-miR-6813-5p & 32 \\
hsa-miR-6782-5p & 28 \\
hsa-miR-6805-5p & 28 \\
hsa-miR-6132 & 27 \\
hsa-miR-6085 & 21 \\
hsa-miR-3937 & 15 \\
hsa-miR-3180 & 14 \\
\hline
\end{tabular}

miRNA mainly performs its regulatory function through its targets. Functions of these predicted target genes significantly enriched in the processes related with metabolic, biosynthetic, transcriptional and protein modification etc., biological regulatory mechanisms were integrated in several main KEGG pathways, most of which are cancer-related pathways, such as Ras signaling pathway, Hippo signaling pathway, ErbB signaling pathway, other types of O-glycan biosynthesis etc. These pathways regulate cell proliferation, adhesion, migration, differentiation, evasion and angiogenesis [28-31]. The most significant KEGG pathway was other types of O-glycan biosynthesis. It was believed that the aberrant O-glycan of cell surface influence the adhesion of cancer cells to the endothelium, promoting tumor migration, invasion and favoring cancer cells epithelial-to-mesenchymal transition (EMT). During malignancy progression, tumor cells prevalently stimulate expression of O-glycans that are normally present on embryonic tissues, but not on well-differentiated adult tissues [31,41,42]. This indicates that these dysregulated miRNAs and their target genes may be involving in the tumorigenesis, drug resistance and tumor progression of the insulin resistant HCC cell line.

We also constructed a network which connected the differentially expressed miRNAs to their target genes, also to GO and KEGG enrichment. The network revealed the biological functions and contributed to better understanding of the role of dysregulated miRNAs. In these networks, certain miRNAs functioned as network hubs, such as miR641, miR-6870-5p, miR-7111-5p, miR-4505, miR-4492, and miR-5195-3p. Among these miRNAs, miR-641 was one of the most active miRNAs in the network which targeting 52 genes, 114 GO terms and 44 KEGG pathways. In accord with previous reports, miR-641 was confirmed down-regulated in our present study, suggesting that it might be related with impaired drug sensitivity, migration and adhesion in insulin resistant HepG2 cells. Additional studies are required to elucidate the detailed role of miR-641 in insulin resistant HCC.

Table 7. Crucial KEGGs in the miRNA-KEGG network (degree $>30$ )

\begin{tabular}{llll}
\hline KEGG ID & KEGG_Term & Degree & P value \\
\hline hsa04010 & MAPK signaling pathway & 97 & 0.008968411 \\
hsa04014 & Ras signaling pathway & 87 & 0.003145657 \\
hsa04144 & Endocytosis & 85 & 0.004307909 \\
hsa04015 & Rap1 signaling pathway & 84 & 0.0045072 \\
hsa04024 & cAMP signaling pathway & 76 & 0.035393834 \\
hsa05205 & Proteoglycans in cancer & 73 & 0.000299322 \\
hsa04921 & Oxytocin signaling pathway & 68 & 0.002905436 \\
hsa04261 & Adrenergic signaling in cardiomyocytes & 65 & 0.000450147 \\
hsa04390 & Hippo signaling pathway & 61 & 0.003145657 \\
hsa04360 & Axon guidance & 56 & 0.008176788 \\
hsa04550 & Signaling pathways regulating pluripotency of stem cells & 56 & 0.006210047 \\
hsa04514 & Cell adhesion molecules (CAMs) & 56 & 0.001916405 \\
hsa04728 & Dopaminergic synapse & 54 & 0.0194252 \\
hsa04152 & AMPK signaling pathway & 53 & 0.00777182 \\
hsa04611 & Platelet activation & 51 & 0.017005802 \\
hsa04919 & Thyroid hormone signaling pathway & 49 & 0.035095754 \\
hsa04724 & Glutamatergic synapse & 48 & 0.003145657 \\
hsa04668 & TNF signaling pathway & 44 & 0.031542367 \\
hsa04915 & Estrogen signaling pathway & 42 & 0.001916405 \\
hsa04723 & Retrograde endocannabinoid signaling & 41 & 0.01974073 \\
\hline
\end{tabular}




\begin{tabular}{llll}
\hline KEGG ID & KEGG_Term & Degree & P value \\
\hline hsa04750 & Inflammatory mediator regulation of TRP channels & 41 & 0.01974073 \\
hsa04670 & Leukocyte transendothelial migration & 40 & 0.044378808 \\
hsa04012 & ErbB signaling pathway & 40 & 0.002905436 \\
hsa04666 & Fc gamma R-mediated phagocytosis & 39 & 0.034514602 \\
hsa05414 & Dilated cardiomyopathy & 35 & 0.044378808 \\
hsa04540 & Gap junction & 34 & 0.029084915 \\
hsa04720 & Long-term potentiation & 32 & 0.0110171 \\
hsa04350 & TGF-beta signaling pathway & 31 & 0.0045072 \\
\hline
\end{tabular}

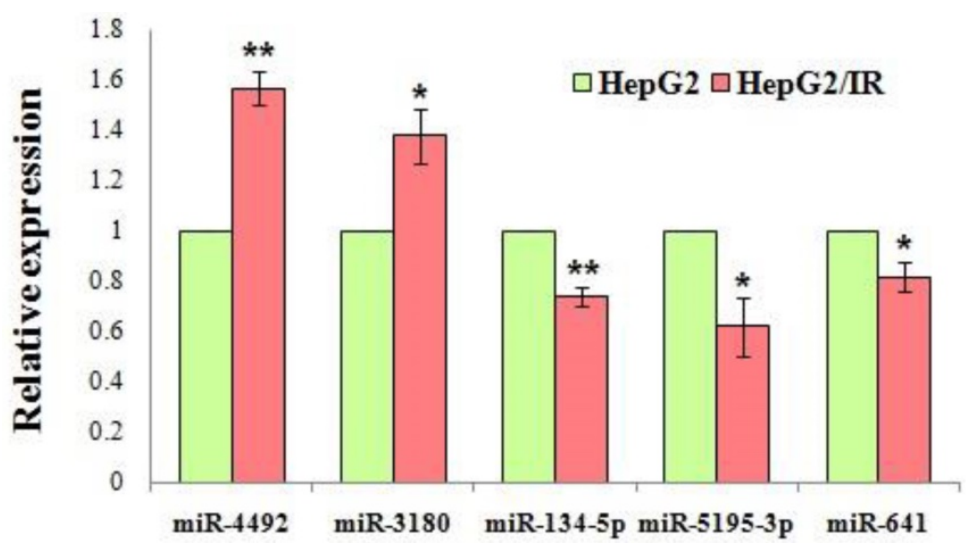

Figure 8. Validation of the microarray results by qRT-PCR. Expression level of the dysregulated miRNAs were shown in the figure. All miRNAs exhibited statistically significant changes in the HepG2/IR cells compared with its parental cells. Relative expression levels were calculated by $2^{-{ }^{\Delta}}{ }_{\mathrm{Ct}}$ method after the (cycle threshold) $\mathrm{Ct}_{\mathrm{t}}$ value (power amplification knee point) was obtained. Experiments were repeated three times with similar results, and the results are presented as mean \pm SD of triplicate experiments. $* \mathrm{P}<0.05, * * \mathrm{P}<0.01$.

The miRNA networks analysis also revealed a range of hub genes (such as KSR2, SYNGAP1), crucial KEGG pathways (Ras signaling pathway, MAPK signaling pathway), and crucial GO (Cellular nitrogen compound metabolic process, Biosynthetic process, Biological process) and most of which were involved in tumorigenesis, drug resistance and poor prognosis of cancer patients [43-48]. Kinase Suppressor of Ras 2 (KSR2) is a molecular scaffold that regulate the intensity and duration of the Ras/Raf/MEK/ERK/ MAPK kinase cascade to facilitate energy consumption and expenditure $[49,50]$. Deletion of KSR2 leads to impair the oxidation of fatty acids and increase their storage as triglycerides, reduced basal metabolic rate thus contributing to obesity and insulin resistance $[49,51]$. KSR2 was also reported as an activator to stimulate tumor cell transformation [45]. Ojha R et al declared that it interacts with the endoplasmic reticulum (ER) stress chaperone (GRP78) and facilitated ER translocation which drove therapy resistance in BRAF-Mutant melanoma [46]. Our present study revealed that as a predicted target gene KSR2 was regulated by eight miRNAs including 7 up-regulated miRNAs (miR-4492, miR-4505, miR-6132, miR-67795p, miR-6780b-5p, miR-6870-5p, miR-7111-5p) and 1 down-regulated miRNA (miR-641). It was involved in many GO categories such as cellular protein modification process, cellular_component, cytosol, ion binding, molecular_function, cellular protein modification process, and participated in Ras signaling KEGG pathway. In our previous study [6-8], we found that insulin resistance contributes to multidrug resistance in HCC cells via activation of the ER stress, suggesting that KSR2 may be involved in therapy resistance in insulin resistant HCC. The expression of KSR2 and its relationship with correlated miRNAs need to be further validated.

The cytosolic protein SYNGAP1/RASA5 (SYNaptic GTPase Activating Protein 1) [48,52] encoded by SYNGAP1 gene was reported as a Ras signaling suppressor comprised in RASA subfamily (Ras GAPs) [53]. It was known that Ras GAPs can inactivate RAS signaling and inhibit oncogenic transformation initiated by RAS. Suppression of Ras GAPs may constitute an additional mechanism whereby aberrant Ras activation promotes tumorigenesis [47,54]. Li L et al [48] declared that expression of SYNGAP1/RASA5 inhibited tumor cell migration/ invasion and growth in mouse model, functioning as a tumor suppressor. Conversely, knockdown of SYNGAP1/RASA5 enhanced Ras signaling to promote tumor cell growth. SYNGAP1/RASA5 also inhibited EMT through regulating actin reorganization. Thus, epigenetic inactivation of SYNGAP1/ RASA5 contributing to hyperactive RAS signaling is involved in Ras-driven human oncogenesis. In our current study, SYNGAP1 was predicted being targeted only by up-regulated miRNAs including miR-4492, 
miR-3180, miR-4505, miR-6085, miR-6795-5p, miR$6805-5 p$, miR-6870-5p miR-7111-5p. It was involved in two GO categories (cellular_component and molecular_function) and participated in Ras signaling pathway. The results suggested that SYNGAP1 might be reduced expression in insulin resistant HepG2 cells. As we known, Ras signaling pathway is often deregulated in tumors through inactivation of Ras inhibitors, SYNGAP1 acts as a tumor suppressor negatively regulated the Ras signaling pathway in cancer. Decreasing expression of SYNGAP1 indicated the enhanced migration, invasion and multidrug resistance in the insulin resistant HCC. Further study should be addressed to validate the expression, regulatory miRNAs, and function of SYNGAP1 in insulin resistant HCC.

In conclusion, our study compared the miRNA expression profile in the insulin resistant HCC cells with its parental cells and identified the differentially expressed miRNAs, which provides information for further understanding of the molecular mechanisms of insulin resistance HCC cells in tumor progression and drug resistance.

\section{Supplementary Material}

Supplementary tables.

http://www.medsci.org/v17p0664s1.zip

\section{Acknowledgements}

This work was supported by the National Natural Science Foundation of China (81602622), Internationally Technological Cooperation Project of Gansu Province (18YF1WA117), Scientific Research Project of Gansu Medical and Health Industry (GSWSKY2016-14), Cuiying Scientific and Technological Innovation Program of Lanzhou University Second Hospital (CY2018-MS11), the Fundamental Research Funds for the Central Universities (lzujbky-2017-81).

\section{Contribution}

L.J. conceived and designed the experiments. L.Z., M.L, L.L. and Y.J. performed the experiments. C.Y., D.F, L.J. and P.H. analyzed the data. L.J., C.Y. and L.L. wrote the paper. All authors read and approved the final manuscript.

\section{Competing Interests}

The authors have declared that no competing interest exists.

\section{References}

1. Siegel R L, Miller K D, Jemal A. Cancer statistics. CA: a cancer journal for clinicians, 2015, 65(1): 5-29.
2. Chenivesse $X$, Franco D, Bréchot C. MDR1 (multidrug resistance) gene expression in human primary liver cancer and cirrhosis[J]. Journal of hepatology, 1993, 18(2): 168-172.

3. Leclercq I A, Morais A D S, Schroyen B, et al. Insulin resistance in hepatocytes and sinusoidal liver cells: mechanisms and consequences[J]. Journal of hepatology, 2007, 47(1): 142-156.

4. Feng Y H, Lin C Y, Huang W T, et al. Diabetes mellitus impairs the response to intra-arterial chemotherapy in hepatocellular carcinoma[J]. Medical Oncology, 2011, 28(4): 1080-1088.

5. Mantovani A, Targher G. Type 2 diabetes mellitus and risk of hepatocellular carcinoma: spotlight on nonalcoholic fatty liver disease[J]. Annals of translational medicine. 2017;5(13).

6. $\mathrm{Li} \mathrm{L}, \mathrm{Li} \mathrm{G}$, Wei $\mathrm{H}$, et al. The endoplasmic reticulum stress response is associated with insulin resistance-mediated drug resistance in HepG2 cells[J]. Neoplasma, 2015, 62(2): 180-190.

7. Liu X, Li L, Li J, et al. Insulin resistance contributes to multidrug resistance in HepG2 cells via activation of the PERK signaling pathway and upregulation of Bcl-2 and P-gp[J]. Oncology reports, 2016, 35(5): 3018-3024.

8. Li L, Liu X, Zhou L, et al. Autophagy Plays a Critical Role in Insulin Resistance-Mediated Chemoresistance in Hepatocellular Carcinoma Cells by Regulating the ER Stress[J]. Journal of Cancer, 2018, 9(23): 4314.

9. $\mathrm{Li} \mathrm{L} \mathrm{J,} \mathrm{Li} \mathrm{G} \mathrm{D,} \mathrm{Wei} \mathrm{H} \mathrm{L,} \mathrm{et} \mathrm{al.} \mathrm{Insulin} \mathrm{resistance} \mathrm{reduces} \mathrm{sensitivity} \mathrm{to}$ Cis-platinum and promotes adhesion, migration and invasion in HepG2 cells[J]. Asian Pac J Cancer Prev, 2014, 15(7): 3123-3128.

10. Oliveto S, Mancino M, Manfrini N, et al. Role of microRNAs in translation regulation and cancer[J]. World journal of biological chemistry, 2017, 8(1): 45.

11. Lu J, Getz G, Miska E A, et al. MicroRNA expression profiles classify human cancers[J]. nature, 2005, 435(7043): 834

12. Fojo T. Multiple paths to a drug resistance phenotype: mutations, translocations, deletions and amplification of coding genes or promoter regions, epigenetic changes and microRNAs[J]. Drug resistance updates, 2007, 10(1-2): 59-67.

13. Allen K E, Weiss G J. Resistance may not be futile: microRNA biomarkers for chemoresistance and potential therapeutics[J]. Molecular cancer therapeutics, 2010, 9(12): 3126-3136.

14. Kabir T D, Ganda C, Brown R M, et al. A microRNA-7/growth arrest specific 6/TYRO3 axis regulates the growth and invasiveness of sorafenib-resistant cells in human hepatocellular carcinoma[J]. Hepatology, 2018, 67(1): 216-231.

15. Xia H, Ooi L L P J, Hui K M. MicroRNA-216a/217-induced epithelialmesenchymal transition targets PTEN and SMAD7 to promote drug resistance and recurrence of liver cancer[J]. Hepatology, 2013, 58(2): 629-641.

16. Meng W, Tai Y, Zhao H, et al. Downregulation of miR-33a-5p in hepatocellular carcinoma: a possible mechanism for chemotherapy resistance[J]. Medical science monitor: international medical journal of experimental and clinical research, 2017, 23: 1295.

17. Yang X, Yin J, Yu J, et al. miRNA-195 sensitizes human hepatocellular carcinoma cells to 5-FU by targeting BCL-w[J]. Oncology reports, 2012, 27(1): 250-257.

18. Long L, Xiang H, Liu J, et al. ZEB1 mediates doxorubicin (Dox) resistance and mesenchymal characteristics of hepatocarcinoma cells[J]. Experimental and molecular pathology, 2019, 106: 116-122.

19. Vlachos I S, Zagganas K, Paraskevopoulou M D, et al. DIANA-miRPath v3. 0: deciphering microRNA function with experimental support[J]. Nucleic acids research, 2015, 43(W1): W460-W466.

20. Czech M P. Insulin action and resistance in obesity and type 2 diabetes[J]. Nature medicine, 2017, 23(7): 804-814.

21. Ratziu V, Goodman Z, Sanyal A. Current efforts and trends in the treatment of NASH[J]. Journal of hepatology, 2015, 62(1): S65-S75.

22. Ahmed A, Wong R J, Harrison S A. Nonalcoholic fatty liver disease review: diagnosis, treatment, and outcomes[J]. Clinical Gastroenterology and Hepatology, 2015, 13(12): 2062-2070.

23. Castera L, Vilgrain V, Angulo P. Noninvasive evaluation of NAFLD[J]. Nature reviews Gastroenterology \& hepatology, 2013, 10(11): 666.

24. Haas J T, Francque S, Staels B. Pathophysiology and mechanisms of nonalcoholic fatty liver disease[J]. Annual review of physiology, 2016, 78: 181-205.

25. Moschen A R, Kaser S, Tilg H. Non-alcoholic steatohepatitis: a microbiota-driven disease[J]. Trends in Endocrinology \& Metabolism, 2013, 24(11): 537-545.

26. Giovannucci E, Harlan D M, Archer M C, et al. Diabetes and cancer: a consensus report[J]. CA: a cancer journal for clinicians, 2010; 60(4): 207-221.

27. El-Serag H B, Hampel H, Javadi F. The association between diabetes and hepatocellular carcinoma: a systematic review of epidemiologic evidence[J]. Clinical Gastroenterology and Hepatology, 2006; 4(3): 369-380.

28. Coggins G E, Farrel A, Rathi K S, et al. YAP1 mediates resistance to MEK1/2 inhibition in neuroblastomas with hyperactivated RAS signalling[J]. Cancer Research, 2019.

29. Ma X, Lu J Y, Moraru A, et al. A novel regulator of ER Ca 2+ drives Hippo-mediated tumorigenesis[J]. Oncogene, 2019: 1-10.

30. Ghaemi Z, Mowla S J. MicroRNA-326 functions as a tumor suppressor in breast cancer by targeting ErbB/PI3K signaling pathway[J]. Frontiers in oncology, 2019; 9: 653.

31. Thomas D, Sagar S, Caffrey T, et al. Truncated O-glycans promote epithelialto-mesenchymal transition and stemness properties of pancreatic cancer cells[J]. Journal of cellular and molecular medicine, 2019; 23(10): 6885-6896. 
32. Liu $\mathrm{Q}, \mathrm{Wu} \mathrm{Y}, \mathrm{Xiao} J$, et al. Long non-coding RNA Prostate Cancer-Associated Transcript 7 (PCAT7) induces poor prognosis and promotes tumorigenesis by inhibiting miR-134-5p in Non-Small-Cell Lung (NSCLC)[J]. Medical science monitor: international medical journal of experimental and clinical research, 2017;23: 6089.

33. Chi J, Liu T, Shi C, et al. Long non-coding RNA LUCAT1 promotes proliferation and invasion in gastric cancer by regulating miR-134-5p/YWHAZ axis[J]. Biomedicine \& Pharmacotherapy, 2019; 118: 109201.

34. Liu Y, Tao Z, Qu J, et al. Long non-coding RNA PCAT7 regulates ELF2 signaling through inhibition of miR-134-5p in nasopharyngeal carcinoma[J]. Biochemical and biophysical research communications, 2017; 491(2): 374-381.

35. Fu D, Lu C, Qu X, et al. LncRNA TTN-AS1 regulates osteosarcoma cell apoptosis and drug resistance via the miR-134-5p/MBTD1 axis[J]. Aging, 2019; 11.

36. Kong Q, Shu N, Li J, et al. miR-641 functions as a tumor suppressor by targeting MDM2 in human lung cancer[J]. Oncology Research Featuring Preclinical and Clinical Cancer Therapeutics, 2018; 26(5): 735-741.

37. Yao R, Zheng H, Wu L, et al. miRNA-641 inhibits the proliferation, migration, and invasion and induces apoptosis of cervical cancer cells by directly targeting ZEB1[J]. OncoTargets and therapy, 2018, 11: 8965.

38. Hinske L C, Heyn J, Hübner M, et al. Intronic miRNA-641 controls its host Gene's pathway PI3K/AKT and this relationship is dysfunctional in glioblastoma multiforme[J]. Biochemical and biophysical research communications, 2017; 489(4): 477-483.

39. Chen J, Cui J, Guo X, et al. Increased expression of miR-641 contributes to erlotinib resistance in non-small-cell lung cancer cells by targeting NF 1[J]. Cancer medicine, 2018; 7(4): 1394-1403.

40. Yan L, Liu J, Zhao Y, et al. Suppression of miR-628-3p and miR-641 is involved in rifampin-mediated CYP3A4 induction in HepaRG cells[J]. Pharmacogenomics, 2017;18(1): 57-64.

41. Tsai C H, Tzeng S F, Chao T K, et al. Metastatic progression of prostate cancer is mediated by autonomous binding of galectin-4-O-glycan to cancer cells[J]. Cancer research, 2016;76(19): 5756-5767.

42. Häuselmann I, Borsig L. Altered tumor-cell glycosylation promotes metastasis[J]. Frontiers in oncology, 2014;4: 28.

43. McCubrey J A, Steelman L S, Chappell W H, et al. Roles of the Raf/MEK/ERK pathway in cell growth, malignant transformation and drug resistance[J]. Biochimica et Biophysica Acta (BBA)-Molecular Cell Research, 2007; 1773(8): 1263-1284.

44. McKay M M, Morrison D K. Integrating signals from RTKs to ERK/MAPK[J]. Oncogene, 2007; 26(22): 3113.

45. Fernandez M R, Henry M L D, Lewis R E. Kinase suppressor of Ras 2 (KSR2) regulates tumor cell transformation via AMPK[J]. Molecular and cellular biology, 2012; 32(18): 3718-3731.

46. Ojha R, Leli N M, Onorati A, et al. ER Translocation of the MAPK Pathway Drives Therapy Resistance in BRAF-Mutant Melanoma[J]. Cancer discovery, 2019; 9(3): 396-415.

47. Grewal T, Koese M, Tebar F, et al. Differential regulation of RasGAPs in cancer[J]. Genes \& cancer, 2011; 2(3): 288-297.

48. Li L, Fan Y, Huang X, et al. Tumor Suppression of Ras GTPase-Activating Protein RASA5 through Antagonizing Ras Signaling Perturbation in Carcinomas[J]. iScience, 2019;21: 1.

49. Costanzo-Garvey D L, Pfluger P T, Dougherty M K, et al. KSR2 is an essential regulator of AMP kinase, energy expenditure, and insulin sensitivity[J]. Cell metabolism, 2009; 10(5): 366-378.

50. Dougherty M K, Ritt D A, Zhou M, et al. KSR2 is a calcineurin substrate that promotes ERK cascade activation in response to calcium signals[J]. Molecular cell. 2009; 34(6): 652-662.

51. Pearce LR, Atanassova N, Banton M C, et al. KSR2 mutations are associated with obesity, insulin resistance, and impaired cellular fuel oxidation[J]. Cell. 2013; 155(4): 765-777.

52. Walkup W G, Washburn L, Sweredoski M J, et al. Phosphorylation of synaptic GTPase-activating protein (synGAP) by Ca2+/calmodulindependent protein kinase II (CaMKII) and cyclin- dependent kinase 5 (CDK5) alters the ratio of its GAP activity toward Ras and Rap GTPases [J]. J Biol Chem. 2015;290(8):4908-27.

53. Maertens O, Cichowski K. An expanding role for RAS GTPase activating proteins (RAS GAPs) in cancer[J]. Advances in biological regulation. 2014; 55: $1-14$.

54. Bernards A, Settleman J. GAPs in growth factor signalling[J]. Growth factors. 2005;23(2): 143-149.

55. Qiu Z, He G. MicroRNA-134 functions as a tumor suppressor gene in gastriccancer, Am. J. Transl. Res. 2016;8 (10): 4320-4328.

56. Shuang T, Wang M, Zhou $Y$, et al. NF-kappaB1, c-Rel, and ELK1 inhibit miR-134 expression leading to TAB1 upregulation in paclitaxel-resistant human ovarian cancer. Oncotarget. 2017; 8(15): 24853-24868. 